\title{
El informe sobre erosión de bases imponibles y traslación de beneficios de la OCDE: origen e implementación en un marco internacional y globalizado \\ Report on OCDE's tax bases erosion and shifting benefits: origin and implementation within international and global framework
}

\section{FERNANDO SERRANO ANTÓN*}

Resumen: El trabajo propone al lector un análisis de las circunstancias que han dado lugar al Informe de la Organización para la Cooperación y el Desarrollo Económico (OCDE) sobre la erosión de bases imponibles y la traslación de beneficios. En el contexto actual de globalización económica, la falta de coherencia de los sistemas fiscales y el unilateralismo han podido dar lugar a situaciones fiscales asimétricas, en gran parte aprovechadas por las multinacionales. Son también objeto de examen los medios e instrumentos utilizados y propuestos por distintas instituciones internacionales para implementar sus acciones con fuerza legal, en concreto, a través del soft law y su aceptación por parte de los distintos Estados, como método utilizado en la lucha contra la evasión y fraude fiscal.

Palabras clave: impuesto sobre la renta - fiscalidad internacional - medidas antiabuso - convenios de doble imposición internacional - sistema de fuentes del derecho - eliminación de la doble imposición internacional - soft law globalización

Abstract: This work is intended to analyze circumstances leading to OCDE's report on tax bases erosion and shifting benefits. Inconsistency of tax systems and unilateralism in current economic globalization framework might have led to asymmetric tax situations, mostly exploited by multinational companies.

Means and tools used and proposed by several international institutions in order to implement legally binding actions through soft law and acceptance by different countries as method used in the fight against tax avoidance and fraud are also discussed.

Key words: income tax - international taxation - anti-abuse measures double taxation agreements - law sources system - double taxation elimination - soft law - globalization

* Profesor titular de Derecho Financiero y Tributario (catedrático acreditado por la Agencia Nacional de Evaluación de la Calidad y Acteditación, ANECA), Universidad Complutense de Madrid. Correo electrónico: serranoa@der.ucm.es 
CONTENIDO: I. INTRODUCCIÓN.- II. ¿SON LAS MEDIDAS ANTIABUSO EN

EL MARCO INTERNACIONAL ALGO NOVEDOSO?.- III. SOFT LAW Y BEPS.-

IV. EL SOFT LAW Y SU INFLUENCIA EN LA CONCEPCIÓN TRADICIONAL DE LAS FUENTES DEL DERECHO.- V. CONTENIDO DEL PLAN DE ACCIÓN BEPS.VI. BEPS Y PAÍSES NO MIEMBROS DE LA OCDE.

\section{INTRODUCCIÓN}

El contexto globalizado en el que vivimos ha beneficiado, sin duda, a las economías nacionales. La globalización ha marcado el ritmo de dichas economías en los últimos años, pero lo novedoso se encuentra en dos factores: primero, el ritmo de integración de las mismas, el cual ha aumentado significativamente en los últimos años; y, en segundo lugar, la influencia de la globalización en los regímenes fiscales del impuesto sobre sociedades en todos los países.

Ya en la segunda década del siglo XX, la Sociedad de Naciones reconoció que la interacción de los sistemas fiscales nacionales puede producir una doble imposición, con efectos adversos sobre el crecimiento. Los países de todo el mundo están de acuerdo en la necesidad de eliminar la doble imposición y en la conveniencia de lograrlo sobre la base de normas internacionales acordadas que sean claras y predecibles, dando certidumbre tanto a los gobiernos como a las empresas. Por tanto, las normas de tributación internacional son un pilar clave en el apoyo al crecimiento de la economía global.

La globalización ha dado como resultado un cambio de paradigma empresarial, partiendo de modelos operativos específicos para cada país para dar lugar a modelos globales basados en organizaciones de gestión matricial y cadenas de suministro integradas que centralizan varias funciones a nivel regional o global. Además, la importancia creciente del sector servicios dentro de la economía, y de productos digitales que a menudo se suministran por Internet, ha facilitado mucho que las empresas localicen muchas actividades productivas en ubicaciones geográficas distantes de la ubicación física de sus clientes. Estos fenómenos han creado oportunidades para que las multinacionales minimicen su carga tributaria. Esto ha motivado una cierta preocupación entre los ciudadanos, que han visto que estas empresas comparativamente pagan menos impuestos que las personas físicas contribuyentes. Esta situación se ha visto intensificada por los momentos de crisis económica que padecemos y, por ende, los gobiernos se han movilizado, dado que tienen hacer frente a menores ingresos y a mayores costes de cumplimiento.

Además, la erosión de la base imponible y el traslado de beneficios (BEPS, por sus siglas en inglés: base erosion and profit shifting) socavan la integridad del sistema tributario. Y, sociológicamente, tanto el público 
en general como la prensa y algunos contribuyentes consideran que los bajos impuestos declarados por las grandes empresas son injustos.

En la base de este estudio que proponemos, se encuentra la tributación entendida como atributo de la soberanía de los países, lo que provoca y produce una cierta interacción de las normas tributarias internas que en algunos casos puede dar lugar a lagunas, solapamientos y fricciones. Cuando los Estados diseñan sus normas tributarias, normalmente no tienen en cuenta los efectos de las normas de otros países, sencillamente por la soberanía a la que nos referíamos más arriba. Dentro de un sistema nacional, se alcanza la coherencia del sistema tributario mediante un principio de correspondencia: un pago deducible por el deudor suele ser gravable en manos del beneficiario. No existe un principio de coherencia similar a nivel internacional, y esto deja mucho espacio para planificar fiscalmente, aunque es cierto que los Estados han venido cooperando para garantizar la coherencia en un ámbito muy limitado, esto es, para evitar la doble imposición o para luchar contra planificaciones fiscales abusivas.

Como ya se ha indicado, los resultados de la globalización sobre el derecho tributario son visibles en buena parte de los elementos esenciales de los principales impuestos de nuestro sistema tributario (en especial, el impuesto sobre sociedades y el impuesto sobre la renta de las personas físicas, además del impuesto sobre la renta de no residentes). Dichos efectos proceden, fundamentalmente, del «efecto simpatía», al copiar las soluciones dadas en otros ordenamientos tributarios, y de la aplicación de todo tipo de directrices que, en el mejor de los casos, reciben el nombre de pseudo-derecho. Se produce así una situación de «policentrismo de fuentes», entre las que hay que incluir tanto las fuentes tradicionales (hard law) como toda una serie de informes y directrices, que conforman lo que se suele denominar soft law (o «derecho blando») ${ }^{1}$ y que, dada su generalizada aceptación y adopción por los Estados, ha ido paulatinamente adquiriendo el status de semi-hard law o derecho impropio. Y lo relevante es que esto se produce en muchos aspectos, en muchas ocasiones, por una curiosa interacción entre el derecho tributario internacional y el derecho comunitario, o incluso la derivación que de este derecho ha realizado el Tribunal de Justicia de la Unión Europea.

En este contexto, y a falta de que sean aceptadas las propuestas de coordinación fiscal internacional formuladas, por poner solo un ejemplo, en el ámbito de la OCDE, la penetración de los principios y la lógica del derecho tributario internacional en el ámbito interno de los distintos países es creciente.

1 Ver MARTín JIMÉNEZ, Adolfo J. Towards Corporate Tax Harmonization in the European Community. Londres: Kluwer, 1999; ANEIROS PEREIRA, Jaime. "Soft law e interpretación: las directrices de la OCDE en materia de precios de transferencia». Crónica Tributaria, 6 (2011), p. 10. 
Precisamente, esta cooperación internacional en la lucha contra la evasión fiscal se ha visto operativa en los convenios de doble imposición internacional (en adelante, los CDI) y también en determinada normativa interna de los Estados, produciendo los resultados correctos, no dando lugar a la erosión de la base imponible ni al traslado de beneficios. La cooperación internacional ha producido principios compartidos y una red de miles de CDI que se basan en estándares comunes y que, por tanto, evitan generalmente la doble imposición de los beneficios de las actividades transfronterizas. Ahora bien, los CDI y la normativa actual también han revelado debilidades que crean oportunidades para la citada erosión y traslado de beneficios. Dichas debilidades se relacionan sobre todo con ejemplos en los que las diferentes normas impositivas producen una doble exención o una imposición inferior a la ordinaria, con estructuras artificiosas que logran una baja o nula imposición. Creemos, sinceramente, que la baja o nula imposición no deben ser per se causa de preocupación, pero sí deben serlo cuando están asociadas a prácticas que disocian artificiosamente la base imponible de las actividades que la generaron.

Junto con lo anterior, la expansión de la economía digital también supone desafíos para la fiscalidad internacional. La economía digital se caracteriza por basarse en activos intangibles, por el uso masivo de datos (especialmente de datos personales), por la adopción general de modelos comerciales que aprovechan el valor de las externalidades generadas por los servicios gratuitos y, finalmente, por la dificultad de determinar la jurisdicción bajo la que tiene lugar la creación de valor. Es un hecho que las nuevas maneras de hacer negocio pueden producir una reubicación de las funciones comerciales esenciales y, en consecuencia, una diferente distribución de la potestad tributaria. Es importante examinar con cuidado el modo en el que las empresas de la economía digital añaden valor y obtienen beneficios, para determinar si es preciso, y hasta qué punto lo es, adaptar las normas actuales para tener en cuenta las características específicas de esas actividades económicas y evitar la erosión de la base imponible y el traslado de beneficios.

Todas estas debilidades e inconsistencias pueden dar lugar a una serie de situaciones indeseables. Sin embargo, el caldo de cultivo de dichas situaciones está ahí, por lo que cada vez más algunos gobiernos pierden ingresos por impuestos sobre sociedades. A su vez, la competencia fiscal lesiva reaparece y desaparece el consenso o los estándares internacionales por medidas unilaterales. Así pues, en este contexto, aparece el proyecto de la OCDE denominado Informe BEPS', apoyado por el Grupo de los 20 (G20), haciendo hincapié en el multilateralismo como método para

2 OECD (Organisation for Economic Co-operation and Development). Lucha contra la erosión de la base imponible y el traslado de beneficios. OECD Publishing, 2013 (http://www.oecd-ilibrary.org/taxation/ abordando-la-erosion-de-la-base-imponible-y-la-deslocalizacion-de-beneficios_9789264201224-es). 
resolver los problemas planteados. A ese Informe le ha seguido un plan de acción ${ }^{3}$ que: (i) identifica las acciones necesarias para paliar la erosión de la base imponible y el traslado de beneficios; (ii) establece plazos para poner en marcha estas acciones; y (iii) identifica los recursos necesarios y la metodología para poner en marcha estas acciones.

\section{II. ¿SON LAS MEDIDAS ANTIABUSO EN EL MARCO INTERNACIONAL ALGO NOVEDOSO?}

La preocupación de los Estados por la evasión y el fraude fiscal internacional no es nueva. Desde hace varias décadas, la OCDE viene estudiando y publicando informes sobre el uso indebido de los CDI. Ya en 1977, el Comité de Asuntos Fiscales decidió comenzar un análisis sistemático de esta cuestión, constituyendo para ello el Grupo de Trabajo número 8 de dicho Comité.

Posteriormente, el Modelo de Convenio de la OCDE para evitar la doble imposición internacional (en adelante, MC OCDE) de 1992 modificó los apartados 11 a 26 de los comentarios al artículo 1 de dicho Modelo de Convenio, dedicados al «Improper Use of Tax Treaties», basándose en dos informes de 1987, denominados Base Companies Report y Conduit Companies Report ${ }^{4}$, incluyendo una recomendación general para la inclusión en los CDI de las medidas antiabuso que después se analizarán. En el año 2001, la OCDE publicó un documento denominado Draft Contents of the 2002 Update to the Model Tax Convention, cuyas conclusiones fueron incorporadas a la versión del año 2003 de los comentarios al artículo 1 del MC OCDE, en donde se contienen innovadoras propuestas relacionadas con el uso indebido de los CDI que han propiciado un intenso debate tanto en el ámbito académico como en el ámbito profesional.

Más recientemente, las iniciativas tanto de la Unión Europea (UE) (reaccionando frente a la planificación fiscal agresiva $a^{5}$ como de la OCDE frente la erosión de bases imponibles y la deslocalización de beneficios inciden de nuevo en la utilización abusiva de los CDI, ya sea de forma autónoma o bien en combinación con la normativa interna de uno o de ambos Estados contratantes ${ }^{6}$.

3 OECD. Plan de acción contra la erosión de la base imponible y el traslado de beneficios. OECD Publishing, 2014 (http://www.oecd-ilibrary.org/taxation/plan-de-accion-contra-la-erosion-de-la-baseimponible-y-el-traslado-de-beneficios_9789264207813-es).

4 OECD. Model Tax Convention. París: OECD Publishing, 2002, vol. II, pp. I-(5) e I-(6).

5 Ver Comisión Europea. “Comunicación de la comisión al parlamento europeo y al consejo. Plan de acción para reforzar la lucha contra el fraude fiscal y la evasión fiscal. 6.12.2012, COM(2012), 722 final», relativa a la planificación fiscal agresiva (http://ec.europa.eu/taxation_customs/resources/ documents/taxation/tax_fraud_evasion/com_2012_722_es.pdf).

6 Ver OECD. Lucha contra la erosión de la base imponible y el traslado de beneficios; Plan de acción contra la erosión de la base imponible y el traslado de beneficios.

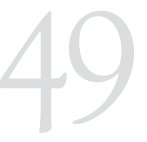

EL INFORME SOBRE EROSIÓN DE BASES IMPONIBLES Y

TRASLACIÓN DE BENEFICIOS DE LA OCDE: ORIGEN E IMPLEMENTACIÓN EN UN MARCO INTERNACIONALY GLOBALIZADO

\section{REPORT ON}

OCDE'S TAX

BASES EROSION

AND SHIFTING

BENEFITS:

ORIGIN AND

IMPLEMENTATION

WITHIN

INTERNATIONAL

AND GLOBAL

FRAMEWORK 
A lo largo de este período, es posible identificar una clara evolución en la postura de la OCDE, abandonándose la visión formalista inicial respecto a la posibilidad de reaccionar contra esquemas elusivos a través de cláusulas antiabuso aprobadas de forma unilateral por los Estados contratantes ${ }^{7}$. Este hecho goza de especial relevancia, ya que, en un primer momento, la posición de la OCDE daba lugar a posiciones divergentes sobre la interpretación, aplicación y alcance de las medidas antiabuso ${ }^{8}$. En todo caso, sí es importante recalcar que la iniciativa BEPS no supone un trabajo radicalmente novedoso, si no que se trata de ideas e informes ya existentes en el marco de la organización internacional, aunque han sido relanzados y cargados de razón por el contexto económico en el que nos hallamos.

Pese a la evolución de la postura de la OCDE en este ámbito, los vigentes párrafos 9.5 y 9.6 de los comentarios al artículo 1 continúan poniendo de manifiesto los problemas que puede plantear, de cara a la aplicación de un CDI, una diferente concepción del término fraude o abuso por los Estados contratantes. Por ello, se advierte que la potencial aplicación de la cláusula genérica antiabuso no implica que no resulte necesario incluir en los propios CDI cláusulas de «beneficiario efectivo» como las habitualmente incluidas en los artículos 10, 11 y 12 o las previsiones antiabuso que contemplan los artículos 13.4 y 17.2 del MC OCDE. Como cabe observar, bajo esta previsión subyace el planteamiento inicial de la OCDE de fijar normas antiabuso armonizadas que impidan a los Estados alterar el reparto de la potestad tributaria inicial a través de la aplicación de normas antiabuso internas.

Es por ello que, a nuestro juicio, y pese a la actual postura de la OCDE, no debe aceptarse sin más la pacífica coexistencia entre las normas antiabuso internas, ya sean estas generales o específicas, y los convenios de doble imposición, debiendo analizarse detenidamente, al margen de la interpretación dinámica que sorprendentemente propugnan los

7 Ver MARTín JIMÉNEZ, Adolfo J. «Las medidas antielusivas/antiabuso internas y los convenios para la eliminación de la doble imposición". En SOLER ROCH, María Teresa y Fernando SERRANO ANTÓN (dirs.). Las medidas antiabuso en la normativa interna española y en lo convenios para evitar la doble imposición internacional. Madrid: IEF, 2002, p. 158.

8 Con ello no quiere decirse que, una vez incorporada la posición de la OCDE a los comentarios al Modelo, desaparezcan instantáneamente todo tipo de dudas respecto a la compatibilidad de las cláusulas antiabuso internas y los CDI que no contemplan su aplicación. A nuestro juicio, es posible identificar una incongruencia en la postura de la OCDE, al mantener un enfoque formalista para las cláusulas antiabuso que tradicionalmente se han venido incluyendo en los CDI (beneficiario efectivo, limitación de beneficios, etc.), abandonando dicha posición cuando se refiere a las cláusulas antiabuso, ya sean estas de carácter general o específico, aprobadas por los Estados miembros. Dicho en otros términos, tras los nuevos comentarios al artículo 1 del MC OCDE, continúan existiendo las mismas dudas con respecto a la posibilidad de aplicar las cláusulas antiabuso de carácter interno a una situación regulada por un CDI, dado que una diferente concepción del término «abuso" podrá dar lugar a una desigual aplicación del tratado por los Estados contratantes, circunstancia que, desde nuestro punto de vista, atentaría contra los principios y fines que lo inspiran. En este sentido, ver ALMUDí CID, José Manuel. El régimen jurídico de transparencia fiscal internacional. Madrid: IEF, 2005, pp. 167ss. 
comentarios al $\mathrm{MC} \mathrm{OCDE}^{9}$, si existe algún tipo de discrepancia entre la cláusula antiabuso que se pretende aplicar y el texto del CDI o el contexto en el que este fue celebrado.

Como reconoce la OCDE, los contribuyentes tienen la posibilidad de aprovechar, al margen de los CDI, las diferencias entre los niveles impositivos existentes en los Estados y los beneficios fiscales reconocidos en las legislaciones fiscales de los diferentes países, correspondiendo a los Estados en cuestión adoptar las disposiciones adecuadas en su legislación interna para evitar tales maniobras ${ }^{10}$. Ahora bien, la validez dichas disposiciones internas estará condicionada a que estas respeten el texto de los CDI celebrados hasta la fecha y su contexto, sin que puedan dar lugar a que la distribución del poder tributario pactado en una norma internacional se vea alterado por mor de la aplicación de normas antiabuso internas.

Sin duda, el relevante crecimiento de la red de CDI de los Estados en los últimos años multiplica las consecuencias de su posible utilización fraudulenta mediante la utilización de construcciones jurídicas artificiosas que permitan a los contribuyentes la obtención tanto de las ventajas fiscales previstas en determinadas legislaciones internas como las reducciones impositivas establecidas en los CDI.

Existen otras muchas causas que dan lugar al caldo de cultivo necesario para que surja el posible abuso de los CDI. En primer lugar, de la interconexión económica mundial ha resultado que la actividad del contribuyente, ya sea persona física o jurídica, tenga repercusión en varias jurisdicciones. En segundo lugar, la propia libertad de circulación de capitales ha favorecido la búsqueda por los contribuyentes de las estructuras de planificación fiscal que más le interesan, basadas en el treaty shopping. En tercer lugar, tampoco pasa desapercibida la fácil constitución de sociedades, con la consiguiente personalidad jurídica y la posibilidad de invocar la aplicación de los beneficios que se incluyen en los CDI. Y, por último, la competencia fiscal entre los Estados ha dado lugar a una suerte de subasta tributaria, en la que se ofrece a los potenciales contribuyentes las mejores condiciones. España no se encuentra al margen de esta situación. De hecho, participa y se beneficia de la misma manera que los Estados de nuestro entorno.

Así pues, las medidas antiabuso contenidas en los CDI surgen como reacción a una serie de comportamientos abusivos de los contribuyentes. Es muy difícil identificar todas las estructuras fiscales que existen para la reducción de la carga fiscal, ya que, en primer lugar, algunos de esos comportamientos son meras economías de opción. Por otro lado,

EL INFORME SOBRE EROSIÓN DE BASES IMPONIBLES Y TRASLACIÓN DE BENEFICIOS DE LA OCDE: ORIGEN E IMPLEMENTACIÓN EN UN MARCO INTERNACIONAL Y GLOBALIZADO

REPORT ON OCDE'S TAX BASES EROSION AND SHIFTING BENEFITS:

ORIGIN AND IMPLEMENTATION WITHIN INTERNATIONAL AND GLOBAL FRAMEWORK 
la realidad va por muy delante en cuanto a la creación de nuevas estructuras, por lo que siempre se estará con unos cuantos «metros de retraso» en cuanto a la reacción legal. En todo caso, se trata de una labor muy casuística, en la que no cabe una posición preestablecida sobre el carácter abusivo de la estructura.

\section{SOFT LAW Y BEPS}

Desde el punto de vista de las organizaciones internacionales, en el ámbito de la UE, la comunicación de la Comisión Europea titulada «Un proyecto para la Unión Europea» ${ }^{11}$ establece específicamente que la regla de la unanimidad en la toma de decisiones en materia fiscal dificulta poder adoptar medidas de política fiscal necesarias para el mejor funcionamiento del mercado interno. En la citada comunicación, la Comisión manifiesta que debe considerarse el uso de instrumentos alternativos que contribuyan a la adopción de las requeridas medidas fiscales a nivel europeo. El uso de los mencionados instrumentos también fue confirmado por el Libro Blanco de la Comisión relativo a la buena gobernanza europea ${ }^{12}$ y, subsecuentemente, en el Acuerdo Interinstitucional Para una Eficaz Elaboración del Derecho Comunitario ${ }^{13}$. En ausencia de un debate relativo a una nueva configuración del derecho comunitario, la Comisión entiende que tanto el derecho originario como el derivado continúan siendo los pilares o ejes sobre los que debe descansar la elaboración del ordenamiento de la UE. Sin embargo, los nuevos instrumentos pseudo-normativos creados deben explorarse y promoverse con el objeto de asegurar la buena gobernanza.

Mucho se ha debatido dentro de la doctrina en torno al origen y noción del soft law. En su origen, el soft law no se refería expresamente al ordenamiento jurídico internacional. Sin embargo, ha sido en esta disciplina que el término fue acogido doctrinalmente y es también en ella que ha dado lugar a amplios debates ${ }^{14}$.

En cuanto a la definición del soft law ${ }^{15}$, hay que partir de la premisa de que no tiene un significado unívoco. Su naturaleza heterodoxa ha llevado a que el término sea definido por la doctrina de manera muy diversa: así, puede ser considerado como una serie de actos que no son legalmente vinculantes pero a los que la práctica les atribuye una fuerza

11 Comisión Europea. «Comunicación de la comisión al parlamento europeo y al consejo. Un proyecto para la Unión Europea. 22-05-2002, COM (2002), 247 final».

12 Comisión Europea. «European Governance. A White Paper. 25-07- 2001, COM (2001), 428».

13 Ver «Inter-institutional Agreement on a Better Law Making». Diario Oficial de la Unión Europea, C $321,31.12 .2003$.

14 MAZUELOS BELLIDO, Ángeles. «Soft law: ¿mucho ruido y pocas nueces?». Revista Electrónica de Estudios Internacionales, 8 (2004); ANEIROS PEREIRA, Jaime. Ob. cit., p. 10.

15 CHINKIN, Christine. «The Challenge of Soft law: Development and Change in International Law». International and Comparative Law Quarterly, 38 (1989). 
moral importante para representar la voluntad política de los Estados miembros de la UE y existir una expectativa de que los Estados miembros harán el máximo posible para seguir con las recomendaciones ${ }^{16}$. Otros entienden que el soft law es una heterodoxa serie de instrumentos que no son fuente del derecho, pero que tienen relevancia en la formación de las normas futuras o en la interpretación de las vigentes ${ }^{17}$. Para terminar con las definiciones dadas, se lo ha considerado como hechos o actos que no pueden ser incluidos como fuentes de derecho, según la definición contenida en el artículo 38 del Estatuto del Tribunal Internacional de Justicia, reconociéndose, por una parte, su carácter residual y, por otra, su falta de valor legal ${ }^{18}$. En todo caso, se puede entender que el apelativo law hace referencia al carácter jurídico de estos actos, de manera que la expresión reivindicaría la existencia de un derecho (law) no vinculante $(\text { soft })^{19}$.

Precisamente, uno de los ámbitos en donde se aprecia una mayor influencia de las recomendaciones de las organizaciones internacionales es en el de las medidas antiabuso y en las operaciones entre empresas participadas o, más propiamente, las operaciones vinculadas o precios de transferencia. En este sentido, debe señalarse que la OCDE publicó en 1995 las Directrices de precios de transferencia (Transfer Pricing Guidelines for Multinational Enterprises and Tax Authorities), las cuales han influido en las distintas legislaciones nacionales. La constante evolución de esta cuestión y la experiencia adquirida durante todo esto tiempo ha ocasionado una revisión de estas directrices en el año $2010^{20}$. No debemos ni podemos dejar de mencionar el núcleo del presente trabajo: el Informe BEPS y su correspondiente plan de acción previamente mencionados, en el que se contiene una acción horizontal sobre precios de transferencia.

También en la Unión Europea se han aprobado modelos de seguimiento y examen de las políticas normativas en materia de precios de transferencia por medio de la resolución del Consejo del 27 de junio de 2006 relativa a un código de conducta sobre la documentación relacionada con los

16 MARTín JimÉnEZ, Adolfo J. Los comentarios al MC OCDE: su incidencia en el sistema de fuentes del derecho tributario sobre los derechos de los contribuyentes. Madrid: CISSPRAXIS, 2003; LAMPREAVE MÁRQUEZ, Patricia. «El soft law y sus implicaciones en la política fiscal de la UE». Crónica Tributaria, 5 (2011), p. 3.

17 FALCÓN Y TELLA, Ramón. «El papel de la jurisprudencia en la armonización fiscal europea». En YEBRA MARTUL-ORTEGA, Perfecto (coord.). Sistema fiscal español y armonización europea. Madrid: Marcial Pons, 1995, pp. 33-54.

18 SACCHETTO, Claudio. «Le fonti del diritto internazionale tributario». En UCKMAR, Victor (coord.). Corso di diritto tributario internazionale. Padua: CEDAM, 2005.

19 NOCETE CORREA, Francisco José. «El diverso alcance del soft law como instrumento interpretativo en la fiscalidad internacional y europea». Crónica Tributaria, 1 (2011), p. 52.

20 OECD. Transfer Pricing Guidelines for Multinationals Enterprises and Tax Administrations. París: OECD Publishing, 2010.

EL INFORME SOBRE EROSIÓN DE BASES IMPONIBLES Y TRASLACIÓN DE BENEFICIOS DE LA OCDE: ORIGEN E IMPLEMENTACIÓN EN UN MARCO INTERNACIONAL Y GLOBALIZADO

REPORT ON OCDE'S TAX BASES EROSION AND SHIFTING BENEFITS:

ORIGIN AND IMPLEMENTATION WITHIN INTERNATIONAL AND GLOBAL FRAMEWORK 
precios de transferencia exigida a las empresas asociadas en la Unión Europea $^{21}$.

En el caso español, esta influencia se ha puesto de manifiesto, sin ninguna duda, en la normativa del impuesto sobre sociedades. La ley 36/2006, del 29 de noviembre, sobre medidas para la prevención del fraude fiscal ha incluido, entre las diversas modificaciones normativas que contempla, la modificación del artículo 16 del Real Decreto Legislativo 4/2004, del 5 de marzo, por el que se aprueba el Texto Refundido de la Ley del Impuesto sobre Sociedades (TRILIS). Con ella se introduce una regla de valoración de las operaciones vinculadas, de acuerdo con cinco métodos admitidos en el contexto internacional, así como la obligación de los sujetos pasivos de documentar estas transacciones. Se recoge, de este modo, el principio de libre concurrencia o Arm's Lengh Principle, así como los aspectos relativos a la documentación de las operaciones realizadas y el importe determinado de acuerdo con el citado principio. Esta última cuestión se traduce, entre otros aspectos, en la creación de una nueva obligación formal de documentación, el llamado masterfile, que implica disponer de una información detallada de los sujetos y de las operaciones realizadas y que ha protagonizado la actualidad normativa y práctica en el ámbito tributario en los últimos tiempos ${ }^{22}$.

En el plano interno, las previsiones que integran la obligación de documentación, aparte de otras cuestiones que desarrollan el régimen jurídico fiscal de los precios de transferencia, han sido objeto de regulación por el Real Decreto 1793/2008, del 3 de noviembre, por el que se desarrolla el Reglamento del Impuesto sobre Sociedades (RIS) y su modificación posterior por el Real Decreto Ley 6/2010, del 9 de abril, así como por el Real Decreto 897/2010, del 9 de julio.

La comprensión de este régimen solo se alcanza si se tiene en cuenta su fuente original. Ello nos obliga a analizar el valor de las Directrices y su trascendencia, pues no debe olvidarse que la compleja regulación que comprenden los textos citados integra el tipo infractor del artículo 16.10 del TRILIS que, como es sabido, prevé un severo sistema de sanciones tributarias para el caso de incumplimiento de esta obligación.

21 Unión Europea. «Directiva sobre precios de transferencia. 28-07- 2006»; «La fiscalidad de las empresas en el mercado interior. 23-10-2001, SEC (2001), 1681»; "Hacia un mercado interior sin obstáculos fiscales. Una estrategia destinada a dotar a las empresas de una base imponible consolidada del impuesto sobre sociedades para sus actividades a escala comunitaria. 23-10-2001, COM (2001), 582 final». Ver, asimismo, RuIBAL PEREIRA, Luz. "Código de conducta para la fiscalidad de las empresas". Noticias de la Unión Europea, 179 (1999), p. 109; CALDERÓN CARRERO, José Manuel. Precios de transferencia e impuesto sobre sociedades. Un análisisde la normativa española desde una perspectiva internacional, comunitaria y constitucional. Valencia: Tirant lo Blanch, 2005, pp. 41 a 43.

22 García-Herrera Blanco, Cristina. «El régimen de documentación de las operaciones vinculadas». Revista Técnica Tributaria, 87 (2009), p. 31. 
La ley 36/2006 de prevención del fraude fiscal citada señala, en su «Exposición de motivos», que uno de los objetivos de la reforma es «adaptar la legislación española en materia de precios de transferencia al contexto internacional, en particular a las Directrices de la OCDE sobre la materia y al Foro Europeo sobre Precios de Transferencia, a cuya luz debe interpretarse la normativa modificada». De hecho, no es infrecuente encontrar referencias a las Directrices sobre precios de transferencia en la normativa de origen interno de los Estados miembros de la OCDE e incluso en las de muchos Estados que no forman parte de esta organización. Por ejemplo, según un estudio elaborado por PricewaterhouseCoopers, algunos de los países miembros que han incluido referencias a las Directrices en su legislación, en reglamentos o en circulares $\mathrm{u}$ otros tipos de instrumentos publicados por la Administración tributaria son Alemania, Australia, Bélgica, Canadá, Dinamarca, Finlandia, Francia, Hungría, México, Noruega, Nueva Zelanda, los Países Bajos, Portugal, Suecia, Turquía y el Reino Unido. Igualmente, entre los Estados no miembros de la OCDE que han incluido este tipo de referencias destacan Ecuador, Kenia, Letonia, Namibia, Rumania, Sudáfrica y Zambia. De todos modos, el contenido de la referencia no es siempre el mismo. Por ejemplo, en algunos casos se indica que la normativa de origen interno se interpretará según las Directrices de la OCDE mientras que en otros se añade que las Directrices tendrán carácter supletorio (Portugal, Nueva Zelanda) o que se tendrán en cuenta a la hora de seleccionar el método más adecuado para aplicar el principio de valoración en condiciones de libre competencia (Letonia) $)^{23}$.

En el caso BEPS, se pretende un proceso inclusivo bajo el liderazgo de la OCDE/G20 y la participación de los países en desarrollo. Es decir, si se quiere que el plan de acción sea operativo, se requiere un proceso global que incluya todas las partes interesadas de cierta importancia. Para ello, y con el fin de facilitar una mayor participación de las principales economías que no son miembros de la OCDE, se ha invitado a los países del G20 interesados que no sean miembros a formar parte del proyecto como «asociados», es decir, en pie de igualdad con los miembros (incluso a nivel de los órganos auxiliares que participan en los trabajos de BEPS). Los países en desarrollo también se enfrentan a problemas relacionados con BEPS, aunque los problemas pueden manifestarse de manera diferente dada la especificidad de sus marcos legales y administrativos. La Organización de las Naciones Unidas (ONU) participa también en el trabajo tributario de la OCDE.

23 PricewaterhouseCoopers. International Transfer Pricing 2009 (http://www.pwc.com/gx/en/ international-transfer-pricing/assets/itp-2009.pdf). Ver VEGA GARCíA, Alberto. “¿Es obligatorio interpretar la normativa española de origen interno sobre precios de transferencia según las directrices de la OCDE? ». Crónica Tributaria, 4 (2011), p. 49. 
Hemos puesto como ejemplo a los precios de transferencia, pero hay muchosotros casos en losquelos Estados hacen suyas las recomendaciones de la OCDE en distintas materias del derecho tributario. Se pueden mencionar, por citar algunos otros ejemplos, el ámbito de las medidas antiabuso del más variado tipo; la delimitación de sus definiciones; la inclusión de medidas ad hoc para determinadas planificaciones fiscales observadas en la arena internacional; la limitación en la aplicación de beneficios fiscales; o el recorte de los beneficios de un CDI en ocasiones especiales, previa determinación por el Comité de Asuntos Fiscales (CFA) de la OCDE. Esto nos obliga a analizar el sistema de fuentes considerando la incidencia de la actuación de la OCDE en la normativa interna de los Estados.

\section{EL SOFT LAW Y SU INFLUENCIA EN LA CONCEPCIÓN TRADICIONAL DE LAS FUENTES DEL DERECHO.}

Como paso previo al estudio de los efectos que tienen las ideas, técnicas y recomendaciones del Informe BEPS, debemos realizar algunas consideraciones sobre el lugar y función que ocupan en las fuentes del derecho. Para ello, debemos analizar, en primer lugar, su origen, que consiste en recomendaciones que emanan de determinadas instituciones supranacionales, en este caso, de la OCDE. Y, en segundo lugar, su posible definición como fuente legal, siendo actos que carecen de eficacia normativa y que son dictados por organismos internacionales, ya tengan estos poder normativo o $\mathrm{no}^{24}$.

Ambos rasgos —el origen y su posición en el sistema de fuentes — son los característicos de este tipo de disposiciones pues, por un lado, carecen de efecto directo y, por otro, tienen su medio ambiente en el ámbito de las organizaciones internacionales. Las denominaciones con las que nos podemos encontrar para referirse a este conjunto de instrumentos son variadas pues, entre otros, se incluyen las recomendaciones, las decisiones, las comunicaciones, las directrices, los códigos de conducta y otras figuras ${ }^{25}$.

La exclusión del sistema de fuentes resulta de las propias normas que regulan esta cuestión, pues ni las normas internacionales ni las normas comunitarias ni las propias normas españolas incluyen a alguno de estos instrumentos entre aquellos que gozan de los efectos jurídicos propios de una norma de tal carácter. Es decir, carecen de la eficacia directa y

24 AlARCÓN GARCíA, Gloria. «El Soft Law y nuestro sistema de fuentes». En AUtORES VARIOS. Tratado sobre la ley general tributaria. Homenaje a Alvaro Rodríguez Bereijo. Cizur Menor: Aranzadi, 2010, pp. 271-298.

25 SARMIENTO, Daniel. «La autoridad del derecho y la naturaleza del soft law». Cuadernos de Derecho Público, 28 (2006), pp. 221-266. 
erga omnes, así como de la oponibilidad propia de las leyes y de las demás normas jurídicas ${ }^{26}$.

Sin embargo, se trata de herramientas técnicas con las que se pretende influir en el derecho positivo de los distintos Estados, estableciendo los principios informadores de las distintas reglamentaciones nacionales, detallando el tratamiento posible de los distintos aspectos del tema o realizando otra serie de consideraciones. En efecto, se trata de establecer el marco conceptual de una determinada materia, así como las líneas maestras de una eventual regulación. Por lo tanto, su relevancia se encuentra en el plano principialista, de denominación o de tendencia ${ }^{27}$.

Así pues, los principales problemas que suscita el uso de este tipo de pseudo-normas derivan de la forma en que estas son producidas y de los medios con los que se han dotado de coercibilidad, al ser aprobadas por un centro de producción normativa externo. Además, la falta de sanción por incumplimiento puede revelar su ausencia de fuerza vinculante. Sin embargo, no compartimos esta última apreciación, al menos con relación a todos los instrumentos de soft law, dado que la falta de cumplimiento puede conllevar una suerte de contramedidas políticas o económicas. El ejemplo más claro en el ámbito de la UE es el Código de Conducta para paliar la competencia desleal entre los Estados miembros de la misma ${ }^{28}$. Muchas son las críticas que ha recibido el código citado, debido a que no puede ser objeto de un control de legalidad, de una interpretación prejudicial (puesto que no existe disposición alguna especial a tal fin), ni puede ser objeto de sanción directa por falta de cumplimiento. El Código estableció un listado de regímenes existentes en los Estados miembros de la UE y sus territorios dependientes o asociados que debían ser derogados por ser contrarios a la competencia leal entre los Estados miembros y por perjudicar, por ello, la correcta marcha del mercado interior. Pese a las múltiples manifestaciones en contra, finalmente no solo se acordó el desmantelar los regímenes penados (conocido como cláusula roll-back) sino que los Estados miembros se comprometieron a no establecer en el futuro regímenes contrarios a los criterios establecidos por la Comisión Europea (conocido como cláusula stand-still). La falta de cumplimiento no quedó impune, dado que del texto se derivan medidas coercitivas, quizás meramente políticas, pero de considerable trascendencia.

De lo anterior queda patente la eficacia de los actos soft law, siendo múltiples los instrumentos elaborados en materia fiscal que han supuesto un avance en lo que a política fiscal de la UE se refiere. Lo que puede sostenerse en contra es la amenaza que acarrea el uso abusivo de este tipo de actos por ciertas instituciones, fundamentalmente la Comisión

EL INFORME SOBRE EROSIÓN DE BASES IMPONIBLES Y TRASLACIÓN DE BENEFICIOS DE LA OCDE: ORIGEN E IMPLEMENTACIÓN EN UN MARCO INTERNACIONAL Y GLOBALIZADO

\section{REPORT ON} OCDE'S TAX BASES EROSION AND SHIFTING BENEFITS:

ORIGIN AND IMPLEMENTATION WITHIN INTERNATIONAL AND GLOBAL FRAMEWORK 
Europea, y el riesgo que subyace en la transformación de actos soft law en hard law sin pasar por las fases establecidas por el ordenamiento de la UE para ello. La Comisión es una institución que carece de toda autoridad para legislar, pudiendo haberse dado la situación, en las actuales circunstancias, de adoptarse actos soft law que nunca se hubieran tramitados como hard law, al haberse opuesto el Parlamento.

La finalidad que se pretende es la de lograr la aproximación de legislaciones y es por ello que su utilización es frecuente en organizaciones supranacionales ${ }^{29}$. Su uso responde en algunos casos, a la falta de poder legislativo — verbigracia OCDE — y, en otros, a la dificultad de aprobar una determinada normativa, o a la existencia de peculiaridades nacionales que hacen difícil el éxito de un régimen jurídico —en muchos casos, la propia $\mathrm{UE}^{30}$ - .

En ocasiones, el soft law termina siendo la base de la regulación de una determinada materia, como ha sucedido con la regulación de las operaciones vinculadas en España. La forma en la que termina convirtiéndose en derechoobliga a su análisis desde unadoble perspectiva: desde el principio de legalidad y desde el de seguridad. Desde el primer enfoque, debe insistirse en la idea del déficit democrático que se pone de manifiesto en el seno de las organizaciones en las que se aprueban estas disposiciones. La composición gubernamental-administrativa, por técnicos o representantes de distintos países y, a veces, de los sectores económicos implicados, hace que no haya ocasión de plantear un debate en los órganos depositarios de la soberanía nacional sobre las distintas alternativas y soluciones ${ }^{31}$. Por tanto, estas pseudo-normas no gozan de la legitimidad democrática, de las posibilidades de debate, de la publicidad y transparencia que ostentan las fuentes normativas clásicas. En otros casos, se puede dar la paradoja de convertir en vinculante una determinada medida que no ha sido tramitada como norma a través de los procedimientos correspondientes, convirtiéndose en una especie de backdoor legislation ${ }^{32}$.

Desde el punto de vista de la seguridad jurídica, la ausencia de una norma jurídica hace que se afecte de pleno a uno de los contenidos

29 AUJEAN, Michel. «European Commision Launches Comprehensive Strategy to Promote Tax Cooordination in the EU». EC Tax Review, 2 (2007), p. 63.

30 GRIBNAU, Hans. «Improving the legitimacy of soft law in EU Tax Law». Intertax, 35, 1 (2007), pp. 30ss.

31 El Foro conjunto UE-OCDE sobre precios de transferencia, por ejemplo, está compuesto por un experto de la Administración tributaria de cada Estado miembro y diez expertos del sector empresarial. Asisten a las reuniones, en calidad de observadores, representantes de los países candidatos a la adhesión y de la Secretaría de la OCDE.

32 Shelton, Dinah. "Law, Non-Law and the Problem of "Soft Law"». En Shelton, Dinah (ed.). Commitment and Compliance: The Role of Non-binding Norms in the International Legal System. Londres: Oxford University Press, 2003, pp. 1-21; ColLADO YURRITA, Miguel Ángel y otros. "Las fuentes del derecho tributario internacional». En CoRdón EZQUeRro, Teodoro (coord.). Manual de fiscalidad internacional, Madrid: IEF, 2007, volumen I; MARÍN JIMÉNEZ, Adolfo. Towards Corporate Tax Harmonization in the European Community, p. 243; NOCETE CORREA, Francisco José, Ob. cit., p. 52. 
fundamentales de este principio: a saber, el que exista una norma pública, clara y precisa ${ }^{33}$.

En la práctica, este sistema impulsado por las organizaciones internacionalesimplicala homogeneización internacionaldelalegislación y ello supone, al mismo tiempo, un apoyo a la internacionalización de las actividades económicas. Es por ello que se ha llegado a decir que el Informe BEPS es el régimen internacional sobre medidas antiabuso, en la medida en que presumiblemente conseguirá un amplio consenso de los regímenes fiscales. Esta idea subyace en distintos informes de la OCDE, en los que se mantiene que sus informes representan principios internacionalmente aceptados para luchar contra la erosión de bases imponibles y traslado de beneficios. Desde el punto de vista de los sistemas interpretativos legales, se ha afirmado que forman parte del contexto a la hora de la interpretación supuestos confusos.

La adopción de un régimen de marcado carácter internacional favorece a las empresas multinacionales al reducir los costes administrativos y de negociación, pero también evita conflictos entre Administraciones tributarias, así como situaciones de abuso de derecho. Ello siempre que la interpretación del soft law sea uniforme en todos los países. En este sentido, el Consejo de la OCDE y los ministros del G20 han recomendado a los gobiernos su aplicación con el objeto de que sea uniforme y se evite la interpretación unilateral del mismo.

Los Estados tienen varias vías para aceptar este enfoque y una de ellas es aceptar las recomendaciones de la OCDE como base, modelo o antecedente ${ }^{34}$. Es aquí donde entendemos que se encuentra su verdadera importancia. Se trata de elementos a tener en cuenta en la interpretación de las distintas disposiciones nacionales, pues constituyen el antecedente histórico de los preceptos que integran el derecho positivo. Por lo tanto, si las normas tienen que interpretarse de acuerdo con los criterios del artículo 3 del Código Civil, como nos recuerda el artículo 12 de la Ley General Tributaria, resulta inevitable tener en cuenta que la regulación española tiene su origen en las propuestas y recomendaciones realizadas desde las organizaciones internacionales que venimos señalando, por lo que sería de aplicación el criterio de la literalidad de los términos, así como el método del sentido usual, jurídico o técnico de algunas referencias normativas, o la propia integración de lagunas legales, que vendrá determinado por lo señalado en los informes.

A ello hay que añadir el valor que puede concederse al soft law en la interpretación teleológica de los preceptos vigentes pues, en muchos

33 GARCíA NovoA, César. El principio de seguridad jurídica en materia tributaria. Madrid: Marcial Pons, 2000, pp. 127ss.; Aneiros Pereira, Jaime. Ob. cit., p. 8.

34 CALDERÓn CARRERo, José Manuel. «The OECD Transfer Pricing Guidelines as a Source of Tax Law: Is Globalization Reaching the Tax Law? ». Intertax, 35, 1 (2007), nota 6, p. 16.

EL INFORME SOBRE EROSIÓN DE BASES IMPONIBLES Y TRASLACIÓN DE BENEFICIOS DE LA OCDE: ORIGEN E IMPLEMENTACIÓN EN UN MARCO INTERNACIONALY GLOBALIZADO

REPORT ON OCDE'S TAX BASES EROSION AND SHIFTING BENEFITS:

ORIGIN AND IMPLEMENTATION WITHIN

INTERNATIONAL AND GLOBAL FRAMEWORK 
casos, la finalidad de la norma encontrará su explicación en estos textos. En todo caso, su valor interpretativo estará condicionado a que el derecho positivo no contradiga expresamente su literalidad y su sentido.

Estamos seguros de que las conclusiones alcanzadas se verán materializadas en las distintas normas que el legislador español incorpore a nuestro ordenamiento. Es así que a la hora de plantearse distintos supuestos jurídicos, esto es, a la hora de interpretar la normativa, deben de tenerse en cuenta los criterios, principios y soluciones planteados en dichos textos internacionales ${ }^{35}$.

Con relación al estatus jurídico de los instrumentos aprobados por la OCDE, de acuerdo con el artículo 5 de su tratado constitutivo, puede dictar decisiones que serán vinculantes para todos los Estados miembros, excepto si se acuerda lo contrario, así como efectuar recomendaciones y celebrar acuerdos con Estados miembros, con Estados no miembros y con organizaciones internacionales. Así pues, puede observase que no existe ningún inconveniente para que dicte normas jurídicas que creen obligaciones para sus Estados miembros. Sin embargo, en el ámbito de la fiscalidad internacional, la OCDE ha preferido actuar mediante recomendaciones.

Dado que las recomendaciones no generan obligaciones jurídicas para los Estadosmiembros, puede afirmarsequelasDirectrices nosonjurídicamente vinculantes y, por lo tanto, los Estados no están obligados a seguirlas ${ }^{36}$ Sin embargo, y como ha sido advertido previamente, el hecho de que en principio las recomendaciones de las organizaciones internacionales no sean jurídicamente vinculantes no quiere decir necesariamente que no puedan tener ningún efecto con relevancia jurídica ${ }^{37}$. Así ocurre, por ejemplo, en la influencia de las recomendaciones en las normas consuetudinarias del derecho internacional. En efecto, como es sabido, para que surja una norma de este tipo la doctrina suele requerir la concurrencia de un elemento objetivo (la práctica estatal) y de un elemento subjetivo (opinio iuris o convicción sobre su carácter obligatorio). En este sentido, H. Hillgenberg considera que, a pesar de que una recomendación no pueda crear directamente una norma consuetudinaria, sí puede contribuir al surgimiento de la opinio iuris. Más aun, una recomendación también puede promover el surgimiento de una práctica estatal uniforme ya que, como recuerda

35 Hallivis Pelayo, Manuel. «Interpretación de tratados internacionales tributarios». México D.F.: Porrúa, 2011, p. 348; ANEIROS PEREIRA, Jaime. Ob. cit., p. 10.

36 BLOKKER, Niels. "Skating on Thin Ice? On the Law of International Organizations and the Legal Nature of the Commentaries on the OECD Model Tax Convention». En DoumA, Sjoerd y Frank ENGELEN (eds.). The Legal Status of the OECD Commentaries. Ámsterdam: IBFD, 2008, pp. 17-22; VeGa García, Alberto. Ob. cit., p. 49.

37 SCHACHTER, Oscar. «The Twilight Existence of Nonbinding International Agreements». The American Journal of International Law, 71, 2 (1977), p. 301. 
C. Schreuer, las recomendaciones permiten coordinar las prácticas desorganizadas de los Estados ${ }^{38}$.

La posibilidad de que las acciones del programa BEPS (que se discutirán a continuación) hayan dado lugar al surgimiento de derecho internacional o conformen una norma consuetudinaria de este ha sido defendida por autores como $\mathrm{C}$. Thomas, quien considera que pueden darse todos los requisitos para entender que los principios allí contenidos - los estándares internacionales - constituyen una norma consuetudinaria de derecho internacional. En concreto, el autor citado entiende que existe una práctica estatal suficientemente coherente, continuada y general ${ }^{39}$.

Asimismo, en relación con los efectos jurídicos de las recomendaciones de las organizaciones internacionales, debe traerse a colación que estas últimas, a pesar de no ser jurídicamente vinculantes, pueden comportar efectos jurídicos como consecuencia de la aplicación de la doctrina del estoppel o de la noción de aquiescencia. Según esta doctrina, ligada frecuentemente a consideraciones de buena fe, el comportamiento de los Estados debe ser coherente. Por lo tanto, incluso si se aceptara que las operaciones de erosión de bases imponibles o de traslado de beneficios son normas antiabuso, ello no implica que el plan de acción de la OCDE, que regula esta materia de forma detallada, tenga también la condición de norma consuetudinaria ${ }^{40}$.

En el caso de las operaciones entre sociedades o entidades de diferentes Estados, especialmente si ambos son Estados miembros de la OCDE, la aplicación de tales principios de derecho internacional tiene sentido. La razón es que, por consideraciones de buena fe, el hecho de haber votado a favor de la recomendación de seguir las pautas de BEPS puede comportar la necesidad de seguirlas para no perjudicar las expectativas que los otros Estados se hayan podido formar. De todos modos, entendemos que en los supuestos con elementos de internacionalidad es necesario distinguir entre dos tipos de casos: aquellos en los que resulta aplicable un CDI y aquellos en los que no. En el primer tipo de casos, la existencia de un CDI incluyendo normas claras sobre el abuso de un CDI comporta que las recomendaciones de BEPS no interpreten la normativa interna (o por lo menos no únicamente), sino fundamentalmente el artículo o artículos donde se contengan cláusulas específicas antiabuso.

38 HillgenBeRG, Hartmut. "A Fresh Look at Soft Law». European Journal of International Law, 10, 3 (1999), p. 514; SCHREUER, Christoph. «Recommendations and the Traditional Sources of International Law». German Yearbook of International Law, 20 (1977), pp. 103-118, p. 108.

39 THOMAS, Chantal. "Customary International Law and State Taxation of Corporate Income: The Case for the Separate Accounting Method». Berkeley Journal of International Law, 14,1 (1996), p. 130; VEGA GARCía, Alberto. Ob. cit., p. 49.

40 ENGELEN, Frank. "How "acquiescence" and "estoppel" can operate to the effect that the States parties to a tax treaty are legally bound to interpret the treaty in accordance with the commentaries on the OECD Model Tax Convention», en DoumA, Sjoerd y Frank ENGELEN (eds.). Ob. cit, pp. 53-57.

EL INFORME SOBRE EROSIÓN DE BASES IMPONIBLESY TRASLACIÓN DE BENEFICIOS DE LA OCDE: ORIGEN E IMPLEMENTACIÓN EN UN MARCO INTERNACIONAL Y GLOBALIZADO

\section{REPORT ON}

oCDE'S TAX

BASES EROSION

AND SHIFTING

BENEFITS:

ORIGIN AND

IMPLEMENTATION

WITHIN

INTERNATIONAL

AND GLOBAL

FRAMEWORK 
En el segundo tipo de casos, no hay que descartar la posibilidad de aplicar las nociones de aquiescencia y estoppel. Siguiendo el razonamiento de F. Engelen, descrito más arriba, podría considerarse que aquellos Estados que votaron a favor de la recomendación de seguir las recomendaciones de BEPS en la OCDE (a pesar de poderse haber abstenido) posteriormente pueden verse obligados a seguir tales Directrices para no perjudicar las expectativas legítimas de otros Estados. Sin embargo, entendemos que, como sostiene la mayoría de la doctrina en relación con el uso de los comentarios a la hora de interpretar los $\mathrm{CDI}$ en vigor, el hecho de que en principio deba entenderse que las recomendaciones no son vinculantes comporta que si no se indica lo contrario deba continuarse presumiendo tal carácter. En consecuencia, no hay que presuponer que, por el mero hecho de que un Estado haya votado a favor de la recomendación de emplear las recomendaciones BEPS, dicho Estado las vaya a seguir en todos sus aspectos, ya que, al fin y al cabo, se trata de una mera recomendación. Por lo tanto, entendemos que sería muy improbable que un tribunal, si se planteara el caso y en el estado actual de las condiciones, llegara a considerar que un Estado puede tener la legítima expectativa de que los Estados que han votado a favor de la recomendación de seguir la iniciativa BEPS la aplicarán a la hora de configurar o interpretar su normativa interna sobre la materia, de forma que, para evitar un perjuicio al Estado con tal expectativa, el otro deba seguir las recomendaciones BEPS, a pesar de que, en principio, no sean jurídicamente vinculante.

\section{CONTENIDO DEL PLAN DE ACCIÓN BEPS}

El plan de acción se ejecutará en un plazo máximo de dos años, con acciones de 6 meses, 1 año, 15 meses y 2 años. En él se identifican algunas áreas de especial incidencia en la erosión de bases imponibles, tales como la doble exención y los supuestos de exención o baja imposición asociados a prácticas que separen artificiosamente los ingresos imponibles de las actividades que los generaron. Para su solución también se señalan dos instrumentos: la adopción de nuevos enfoques basados en el consenso y la inclusión de disposiciones antiabuso, diseñadas para evitar y contrarrestar la erosión de la base imponible y el traslado de beneficios, asegurando la coherencia del impuesto sobre la renta de sociedades a nivel internacional. En todo caso, parece que se parte de una premisa consistente en que los contribuyentes, en general, y las multinacionales, en particular, defraudan, lo que choca con las orientaciones de una Administración tributaria asistencial, sin recelos y cooperativa (que ha sido la doctrina más utilizada en los últimos años).

La OCDE siempre ha reconocido la gran labor realizada por los CDI para evitar la doble imposición, pero existe la preocupación de que a 
menudo ellos no pueden evitar la doble no imposición que resulta de las interacciones entre dos o más países. En particular, la implicación de terceros países en el marco bilateral formado por socios vinculados por un CDI supone una tensión para las normas existentes, en particular cuando se hace utilizando sociedades instrumentales que tienen poco o ningún fundamento económico en términos de bienes inmuebles, bienes tangibles y empleados afectos a la actividad.

Pasemos a enumerar las acciones previstas en el plan de acción BEPS y su contenido de forma esquemática, sabiendo que cada acción será objeto de análisis por subgrupos que estarán coordinados con otros, dependiendo de la materia.

\section{V.1.Abordar los retos de la economía digital para la imposición}

Se trata de identificar las principales dificultades que plantea la economía digital a la aplicación de las actuales normas impositivas internacionales y desarrollar opciones detalladas para abordar estas dificultades, con un enfoque holístico y considerando tanto la imposición directa como la indirecta. Los temas a examinar incluyen, aunque no se limitan a ello, la capacidad de una compañía de tener una presencia digital significativa en la economía de otro país sin estar sujeta a imposición debido a la falta de un «nexo» de acuerdo con las normas internacionales actuales, la atribución de valor creado a partir de la generación de datos comercializables de ubicación relevante mediante el uso de productos y servicios digitales, la caracterización de los ingresos procedentes de nuevos modelos comerciales, la aplicación de normas relativas a la fuente y el modo de asegurar la recaudación efectiva del IVA/IIC u otros impuestos indirectos relativos al suministro transfronterizo de bienes y servicios digitales.

\section{V.2. Creación de coherencia internacional en el impuesto de sociedades: neutralizar los efectos de mecanismos híbridos}

La globalización implica que las políticas nacionales, incluyendo la política fiscal, no pueden diseñarse aisladamente. La política fiscal es un atributo básico de la soberanía, y cada país tiene el derecho a diseñar su sistema impositivo del modo que considere más adecuado. Al mismo tiempo, la creciente interconexión de las economías nacionales ha puesto de relieve que la interacción entre las normativas fiscales nacionales puede dar lugar a lagunas. Por lo tanto, existe la necesidad de complementar las normas para evitar la doble imposición con un conjunto fundamentalmente nuevo de normas diseñadas para crear una coherencia internacional en el impuesto de sociedades.

EL INFORME SOBRE EROSIÓN DE BASES IMPONIBLES Y TRASLACIÓN DE BENEFICIOS DE LA OCDE: ORIGEN E IMPLEMENTACIÓN EN UN MARCO INTERNACIONAL Y GLOBALIZADO

REPORT ON OCDE'S TAX BASES EROSION AND SHIFTING BENEFITS:

ORIGIN AND IMPLEMENTATION WITHIN INTERNATIONAL AND GLOBAL FRAMEWORK 
El Informe BEPS (OCDE, 2013) reclama el desarrollo de «instrumentos para eliminar o neutralizar los efectos de los mecanismos híbridos y el arbitraje». Los mecanismos híbridos pueden terminar creando una doble imposición no deseada o un aplazamiento a largo plazo de la imposición. Así pues, se trata de desarrollar disposiciones convencionales y recomendaciones para el diseño de normas internas que neutralicen el efecto (por ejemplo, la doble exención, la doble deducción o el diferimiento a largo plazo) de los mecanismos y de las entidades híbridas.

\section{V.3. Refuerzo de las normas sobre las CFC}

Una de las fuentes de preocupación acerca de la erosión de la base imponible y el traslado de beneficios proviene de la posibilidad de crear filiales no residentes y canalizar a través de ellas los ingresos de una empresa residente. Para solventar este problema se han introducido normas sobre la corporación extranjera controlada (CFC, por sus siglas en inglés: controlled foreign corporation) y contra otros tipos de aplazamiento en muchos países. Sin embargo, la normativa sobre CFC de muchos países no siempre combate la erosión de la base imponible y el traslado de beneficios de un modo integral. Además, aunque estas normas en principio suponen la inclusión en el país de residencia de la empresa matriz última, también tienen efectos indirectos favorables en los países de origen porque los contribuyentes no tienen (o tienen menos) incentivos para trasladar los beneficios a una tercera jurisdicción con bajo nivel impositivo.

\section{V.4. Limitar la erosión de la base imponible por vía de deducciones en el interés y otros pagos financieros}

Las deducciones de gastos financieros pueden dar lugar a una doble exención, tanto en situaciones de inversión entrante como saliente. Desde la perspectiva de la inversión entrante, la preocupación relativa a la deducción de los gastos financieros tiene que ver sobre todo con los préstamos por una entidad vinculada y no residente que se beneficia de un régimen fiscal favorable (de baja imposición) para crear excesivas deducciones en forma de interés para el prestatario, sin la correlativa inclusión de los correspondientes ingresos por el prestamista. El resultado es que los pagos por intereses se deducen de la base imponible de las empresas implicadas, mientras que el ingreso goza de un trato favorable o no se grava en absoluto para el cedente del capital, siendo incluso posible que este efecto se produzca aunque el grupo como tal no tenga (o tenga muy poca) deuda con terceros.

Desde la perspectiva de las inversiones salientes, puede que una compañía se endeude para financiar la generación de un ingreso que está exento o que se puede diferir, gozando por lo tanto de una deducción 
corriente por gastos financieros, en tanto que el ingreso correspondiente se aplaza o exonera. Habría que tener en cuenta, por consiguiente, en las normas que regulan la deducción de gastos financieros, que los intereses entre partes vinculadas pueden no estar sometidos a una imposición completa o que la deuda subyacente pueda ser utilizada para reducir inadecuadamente la base imponible del prestatario para generar un diferimiento o incluso una exención de los ingresos. Surgen problemas parecidos cuando consideramos los pagos deducibles por otras transacciones financieras, como las garantías financieras y de rendimientos, instrumentos derivados, seguros cautivos u otro tipo de contratos entre aseguradoras, especialmente en el contexto de los precios de transferencia.

\section{V.5.Combatir las prácticas tributarias perniciosas, teniendo en cuenta la transparencia y la sustancia}

En 1998, la OCDE publicó un informe sobre prácticas tributarias perjudiciales, basado hasta cierto punto en la constatación de que una «carrera de igualación a la baja» se traduciría en última instancia en que los tipos impositivos aplicables a determinadas rentas móviles se igualarían a cero en todos los países, fuera este o no el objetivo de política tributaria que un país determinado tratase de alcanzar. De hecho, acordar un conjunto de reglas comunes podría ayudar a los países a preservar su soberanía en las decisiones en materia tributaria. Las preocupaciones políticas subyacentes expresadas en el informe de 1998 en cuanto a la «carrera de igualación a la baja» en la base del impuesto sobre rentas móviles son tan relevantes hoy como lo fueron hace quince años. Sin embargo, la «carrera de igualación a la baja» a menudo toma hoy en día menos la forma tradicional de regímenes bien delimitados y se produce más en forma de reducciones del tipo impositivo en el impuesto sobre sociedades para determinados tipos de ingresos (tales como ingresos de actividades financieras o de cesión de intangibles).

\section{V.6. Impedir la utilización abusiva de convenios}

La interposición de terceros países en el marco bilateral establecido por los firmantes de un convenio ha propiciado el desarrollo de estructuras tales como establecimientos de empresas extranjeras de baja imposición o de sociedades instrumentales, así como el desplazamiento artificioso de ingresos a través de precios de transferencia. Las cifras de inversión extranjera directa (IED) muestran la magnitud del uso de determinados regímenes para canalizar las inversiones y la financiación intragrupo de un país a otro a través de estructuras de cartera. Con el fin de preservar los efectos previstos en las relaciones bilaterales, las normas deben ser

EL INFORME SOBRE EROSIÓN DE BASES IMPONIBLES Y

TRASLACIÓN DE BENEFICIOS DE LA OCDE: ORIGEN E IMPLEMENTACIÓN EN UN MARCO INTERNACIONALY GLOBALIZADO

REPORT ON OCDE'S TAX BASES EROSION AND SHIFTING BENEFITS:

ORIGIN AND IMPLEMENTATION WITHIN INTERNATIONAL AND GLOBAL FRAMEWORK 
modificadas para abordar el uso de múltiples capas de entidades legales interpuestas entre el país de residencia y el país de la fuente.

El abuso de convenios es una de las fuentes de preocupación sobre la BEPS más importante. El comentario sobre el artículo 1 del Modelo de Convenio de la OCDE ya incluye una serie de ejemplos de disposiciones que podrían utilizarse para abordar situaciones de treaty-shopping, así como otros casos de abuso de convenio que pueden dar lugar a una doble exención. Disponer de cláusulas estrictas contra el abuso de convenios, junto con el ejercicio de la potestad tributaria bajo las leyes internas, son elementos que contribuirán a restaurar la imposición en la fuente en un número de casos.

\section{V.7. Impedir la elusión artificiosa del estatuto de EP}

En muchos países, la interpretación de las normas del convenio sobre establecimientos permanentes (EP) en caso de agentes permite que ciertos contratos para la venta de bienes propiedad de una empresa extranjera sean concluidos dentro del país por vendedores de una filial local de esa empresa extranjera, sin que los beneficios de estas ventas sean imponibles en la misma medida que lo hubieran sido si las ventas se hubieran hecho por un distribuidor local. En ciertos casos, esto ha llevado a las empresas a sustituir los mecanismos en virtud del cual la filial local tradicionalmente actuaba como distribuidor por «mecanismos de comisión», que dan lugar a un desplazamiento de los beneficios fuera del país donde las ventas se llevan a cabo, sin un cambio sustancial en las funciones desempeñadas en ese país. De manera similar, las multinacionales pueden fragmentar artificiosamente sus operaciones entre varias entidades del grupo para caracterizar ciertas actividades como preparatorias y accesorias y así gozar de una excepción a las reglas sobre EP.

\section{V.8. Asegurar que los resultados de los precios de transferencia están en línea con la creación de valor: intangibles, riesgos y capital, y otras transacciones de alto riesgo}

Las normas sobre precios de transferencia sirven para distribuir los ingresos obtenidos por una empresa internacional entre aquellos países en los que la empresa ha hecho negocios. En muchos casos, las normas existentes de precios de transferencia, basadas en el principio de libre concurrencia, distribuyen los ingresos de las multinacionales entre jurisdicciones tributarias de manera eficaz y eficiente. En otros casos, sin embargo, las multinacionales han sido capaces de usar y abusar de las normas para separar el ingreso de las actividades económicas que lo producen y desplazarlo a zonas de baja imposición. Ello se produce 
casi siempre mediante transferencias de intangibles y de otros activos móviles por valores inferiores a su valor real, por la sobre capitalización de aquellas entidades del grupo que tributan a tipos reducidos, y por las asignaciones contractuales de riesgos a territorios de baja imposición, en transacciones que raramente ocurrirían entre partes independientes. En ocasiones se proponen sistemas alternativos para la distribución de los ingresos, incluyendo los sistemas basados en fórmulas. Sin embargo, la importancia de una acción concertada y las dificultades prácticas para lograr un acuerdo sobre el nuevo sistema y para una aplicación consistente en todos los países se traducen en que, más que intentar sustituir el sistema actual de precios de transferencia, la mejor opción es abordar directamente los defectos en el sistema actual, en particular respecto a los retornos relacionados con activos intangibles, riesgo y sobrecapitalización. Sin embargo, algunas medidas especiales, ya sea dentro o fuera de principio de plena concurrencia, pueden resultar necesarias en lo que respecta a activos intangibles, riesgo y exceso de capitalización, con miras a hacer frente a estas deficiencias.

\section{V.9. Establecer metodologías para la recopilación y el análisis de datos sobre la erosión de la base imponible y el traslado de beneficios y sobre las acciones para enfrentarse a ella}

A pesar de los progresos en materia de transparencia que se han conseguido gracias al Foro Global sobre Transparencia e Intercambio de Información, se ha puesto de manifiesto la necesidad de un enfoque más filosófico cuando se trata de impedir la erosión de la base imponible y el traslado de beneficios, lo cual implica más transparencia en diferentes frentes. Para la OCDE es necesario mejorar la recopilación de datos sobre la erosión de la base imponible y el traslado de beneficios. Los contribuyentes deben revelar información más específica acerca de sus estrategias de planificación fiscal, y los requisitos de documentación sobre precios de transferencia deben ser menos onerosos y más específicos.

Es fundamental mejorar la disponibilidad de los datos y el análisis de la erosión de la base imponible y el traslado de beneficios, incluyendo el control de la implementación del plan de acción. El Informe BEPS señala que existen varios estudios y datos que indican que hay una desconexión creciente entre el lugar donde se llevan a cabo las inversiones y las actividades que generan valor y el lugar donde se declaran los beneficios a efectos fiscales. El Informe BEPS señala que hay que seguir trabajando para evaluar este tipo de estudios, para el desarrollo de medidas apropiadas a la escala y efectos de las conductas de erosión de base imponible y de traslado de beneficios, y para controlar el impacto de las medidas adoptadas en el marco del plan de acción

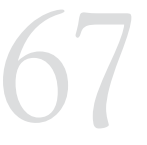

EL INFORME SOBRE EROSIÓN DE BASES IMPONIBLES Y

TRASLACIÓN DE BENEFICIOS DE LA OCDE: ORIGEN E IMPLEMENTACIÓN EN UN MARCO INTERNACIONAL Y GLOBALIZADO

REPORT ON oCDE'S TAX BASES EROSION AND SHIFTING BENEFITS:

ORIGIN AND IMPLEMENTATION WITHIN INTERNATIONAL AND GLOBAL FRAMEWORK 
para tratar de solucionar BEPS. Ello debe incluir técnicas basadas en resultados que busquen medidas de la asignación de ingresos entre jurisdicciones en relación con las medidas de las actividades que generan valor, así como otras técnicas que se pueden utilizar para hacer un seguimiento de los problemas específicos identificados en el plan de acción. En consecuencia, es importante identificar los tipos de datos que los contribuyentes deberían proporcionar a las Administraciones tributarias, así como las metodologías que se pueden utilizar para analizar estos datos y evaluar las posibles consecuencias económicas de los comportamientos y las medidas adoptadas para tratar la erosión de la base imponible y el traslado de beneficios.

\section{V.10. Exigir a los contribuyentes que revelen sus meca- nismos de planificación fiscal agresiva}

Las Administraciones tributarias no disponen, a menudo, de información completa y relevante sobre las estrategias de planificación fiscal. Sin embargo, la disponibilidad de información específica, completa y brindada a tiempo es esencial para permitir que los gobiernos identifiquen rápidamente las áreas de riesgo. Aunque las inspecciones fiscales siguen siendo una fuente clave de información relevante, padecen una serie de limitaciones y carecen de herramientas adecuadas para la detección temprana de las técnicas de planificación fiscal agresiva. Pueden ser útiles, en este sentido, las medidas destinadas a mejorar el flujo de información sobre los riesgos fiscales hacia las Administraciones tributarias y los responsables de la política fiscal («iniciativas de revelación»). Otras medidas potencialmente útiles incluyen programas de cumplimiento cooperativo entre los contribuyentes y las administraciones tributarias, si bien este tipo de iniciativas no han sido exitosas en la gran mayoría de los Estados.

\section{V.11. Reexaminar la documentación sobre precios de transferencia}

Una cuestión clave en la aplicación de los precios de transferencia es la asimetría de información entre los contribuyentes y las Administraciones tributarias. Ello socava potencialmente la aplicación del principio de libre concurrencia. En muchos países, las Administraciones tributarias tienen poca capacidad para desarrollar una visión «panorámica» de la cadena de valor global del contribuyente. Además, las divergencias de enfoque sobre los requisitos de documentación para los precios de transferencia conducen a costes administrativos significativos para las empresas. En ese sentido, es importante que las Administraciones tributarias tengan a su disposición información adecuada acerca de las funciones relevantes 
realizadas por otros miembros del grupo multinacional en materia de servicios intragrupo y otras transacciones.

\section{V.12. Hacer más efectivos los mecanismos de resolución de controversias}

Un complemento importante de la iniciativa BEPS debe ser trabajar para mejorar la eficacia de los procedimientos amistosos. La interpretación y la aplicación de las normas nuevas que resulten del trabajo descrito más arriba introducirán (de hecho ya se producen) elementos de incertidumbre que deberían ser minimizados todo lo posible. Por consiguiente, deben realizarse trabajos destinados a examinar y luchar contra obstáculos que impiden que los países resuelvan las controversias derivadas de la aplicación del convenio mediante los procedimientos amistosos. Asimismo, se debería tener en cuenta la posibilidad de completar las disposiciones existentes sobre les procedimientos amistosos en los convenios fiscales con una cláusula de arbitraje obligatorio y vinculante.

\section{V.13. Desarrollar un instrumento multilateral}

El cumplimiento de las acciones incluidas en el plan de acción sobre la erosión de la base imponible y el traslado de beneficios dará lugar a una serie de resultados. Algunas acciones darán lugar probablemente a recomendaciones relacionadas con disposiciones legales internas, así como a cambios en los Comentarios al MC de la OCDE y a las Directrices de precios de transferencia. Probablemente, otras acciones darán lugar a cambios en el MC de la OCDE. Este es el caso, por ejemplo, de la introducción de una disposición antiabuso de convenio, de los cambios en la definición de EP, de los cambios en las disposiciones sobre precios de transferencia y de la introducción de nuevas disposiciones en el Convenio en relación con los desajustes por mecanismos híbridos. Los cambios en el MC de la OCDE no serán directamente efectivos sin las correspondientes enmiendas de los convenios fiscales bilaterales. Si el proceso se aborda convenio por convenio, el gran número de convenios en vigor puede hacer que este proceso sea muy largo, más aún cuando los países se embarquen en renegociaciones generales de sus convenios fiscales bilaterales. Este es pues la piedra angular de todo el trabajo: icómo incorporar las novedades previstas si existen CDI y no se quiere entrar en la renegociación de los mismos?, iserá posible una convención multilateral?, y ilos contribuyentes aceptarán la entrada en vigor de este tipo de normas sin un aumento de litigiosidad?

EROSIÓN DE BASES IMPONIBLES Y

TRASLACIÓN DE BENEFICIOS DE LA OCDE: ORIGEN E IMPLEMENTACIÓN EN UN MARCO INTERNACIONAL Y GLOBALIZADO

REPORT ON OCDE'S TAX BASES EROSION AND SHIFTING BENEFITS:

ORIGIN AND IMPLEMENTATION WITHIN INTERNATIONAL AND GLOBAL FRAMEWORK 


\section{BEPS Y PAÍSES NO MIEMBROS DE LA OCDE}

Para terminar este trabajo debe analizarse la posición en la que se encuentran los países no miembros de la OCDE. Esta organización, con el objeto de tener el mayor impacto posible, cuenta a efectos del programa BEPS con tres categorías de Estados involucrados: Estados miembros, Estados cooperantes y, finalmente, Estados invitados. Obviamente, no se encuentran representados la totalidad de los países. Así, pues, ipueden resultar afectados países miembros de las Naciones Unidas o de la Comunidad Andina? Desde un punto de vista legal, el programa BEPS no les afectará directamente y aquí se encuentra uno de los puntos débiles de este. En efecto, la falta de unanimidad podría dar lugar a una falla en el sistema total y, por tanto, a afrontar por parte de estos países soluciones unilaterales en la lucha contra la evasión fiscal, no armonizada con el resto de países, lo que podría suponer el fracaso del proyecto.

No debe hablarse de países ganadores o perdedores con relación a BEPS, aunque la realidad confirma que los países pequeños, aquellos otros con una economía basada en materias primas, o países pequeños en los que la prestación de servicios financieros tenga importancia, pueden resultar más contrariados por BEPS.

En todo caso, sí parece ser unánime la opinión de que (aunque se tenga el apoyo político del G20, el Grupo de los 8 y la OCDE), dado el momento económico caracterizado por la crisis, con Administraciones tributarias ávidas de mayor recaudación y la presión de determinados lobbies, ONG y los medios de comunicación, el impacto de la iniciativa BEPS se verá marcado por la mayor o menor determinación de todos los países en la asunción de las acciones descritas más arriba. Sin diálogo y sin consenso entre los países de las distintas regiones, las acciones planteadas no podrán tener éxito. 